\title{
LV myxoma on Real-time 3DTTE in a young patient with dilated cardiomyopathy and apical thrombus
}

\author{
Nguyen Tuan $\mathrm{Vu}^{*}$ \\ Pham Ngoc Thach University of Medicine, MEDIC HCMC, Vietnam
}

\begin{abstract}
A 31 year old man patient presented by dyspnea for one month, no past history of heart disease had been noted. Physical examination detected 3rd gallop sound, sinus tachycardia and LVH were revealed on his ECG. Chest X ray showed a cardiomegaly with enlarged LV arch. Routine 2DTTE demonstrated enlarged LV and severely reduced $\mathrm{EF}$.
\end{abstract}

2DTTE completed by 3DTTE visualized a LV myxoma that was attached to the posterior IVS next to apical area, hypermobile, pedunculated and excrescential. The risk of embolic events is very high. Regarding the severe heart failure, patient was followed up with medical treatment of heart failure and anticoagulant use

\section{Introduction}

LV myxoma is extremely rare disease, surgical resection of myxoma always indicated because of high risk embolic events. LV myxoma may be occasionally detected or by the symptoms caused by embolic events or valvular orifice obstructing. Previously 2DTTE was used to make diagnosis prior to respect the tumor. Currently, 3DTTE overcomes the limitations of 2DTTE in description of shape, size, pedicle, location, relationship with around structure, especially the surface aspect of myxoma.

\section{Case report}

A young man patient of 31 year-old presented at my hospital by heart failure symptoms for one month, including dyspnea on exertion, orthopnea. Physical examination detected an early diastolic gallop sound.

No past history of tobacco, alcohol consumption, systemic hypertension or congenital cardiac defect had been reported. $\mathrm{He}$ was evaluated immediately by a chest $\mathrm{X}$ ray that demonstrated a cardiomegaly with enlargement of the left inferior cardiac arch (Figure 1).

His ECG showed sinus tachycardia and LV hypertrophy with Sokolow-Lyon Index $=65 \mathrm{~mm}$ (Figure 2).

The transthoracic echocardiogrphy initially revealed enlarged LV with $\mathrm{LVDd}=71 \mathrm{~mm}$, severe reduced systolic function of left ventricle: $\mathrm{EF}=20 \%$, the global contraction of $\mathrm{LV}$ diminished severely on parssternal views as well as all apical views (Figure 3).

The two-dimensional echocardiography also recorded an apical thrombus of $13 \mathrm{~mm} \times 9 \mathrm{~mm}$ in diameter that presented as an echogenic mass attached to the apical endocarde (Figures 4 and 5).

The Real-time three-dimensional TTE then have been performed with Live 3D, Full Volume and especially 3D Zoom mode that provided 3D image of the hyperechogenic mass about shape, size, mobility, surface, location very clearly. Based on characteristic findings of the echogenic mass as highly mobile, pediculated, tumor with excrescence aspect, the first diagnosis is LV myxoma (Figures 6-10).

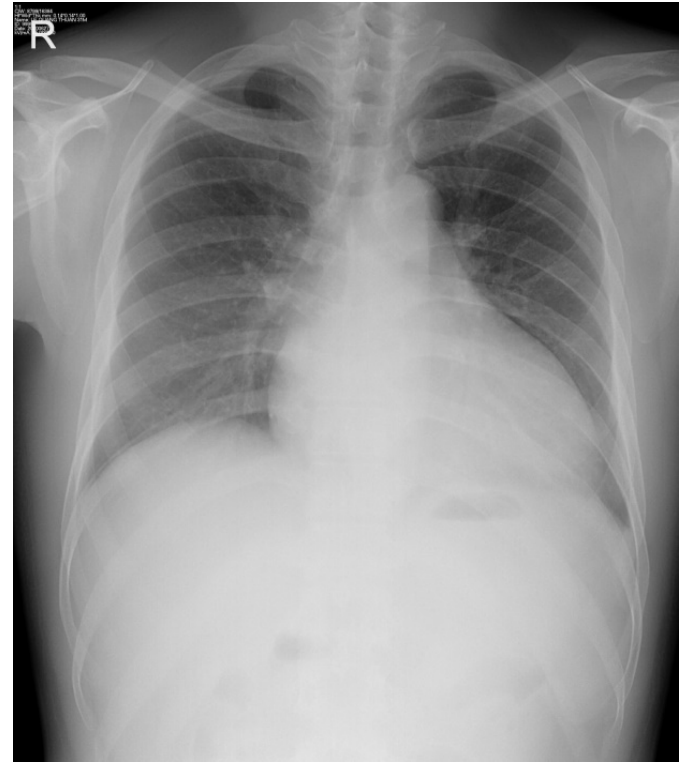

Figure 1. Cardiomegaly with enlarged the left inferior arch (dilated LV)

\section{Discussion}

Primary cardiac tumors are rare, present in less than $0.2 \%$ of unselected autopsy series and associated with high frequency of embolic events. Two most common varieties of these tumors are myxoma and papillary fibroblastoma. Approximately $75 \%$ of all cardiac tumors are

${ }^{\star}$ Correspondence to: $\mathrm{Vu}$ NT, Pham Ngoc Thach University of Medicine, MEDIC HCMC Vietnam, E-mail: tuanvu2401@yahoo.com

Key words: Myxoma, 2DTTE: Two-dimentional Transthoracic Echocardiography, RT-3DTTE: Three-dimensional Transthoracic Echocardiography, LV: Left Ventricle

Received: June 24, 2020; Accepted: June 29, 2020; Published: July 02, 2020 


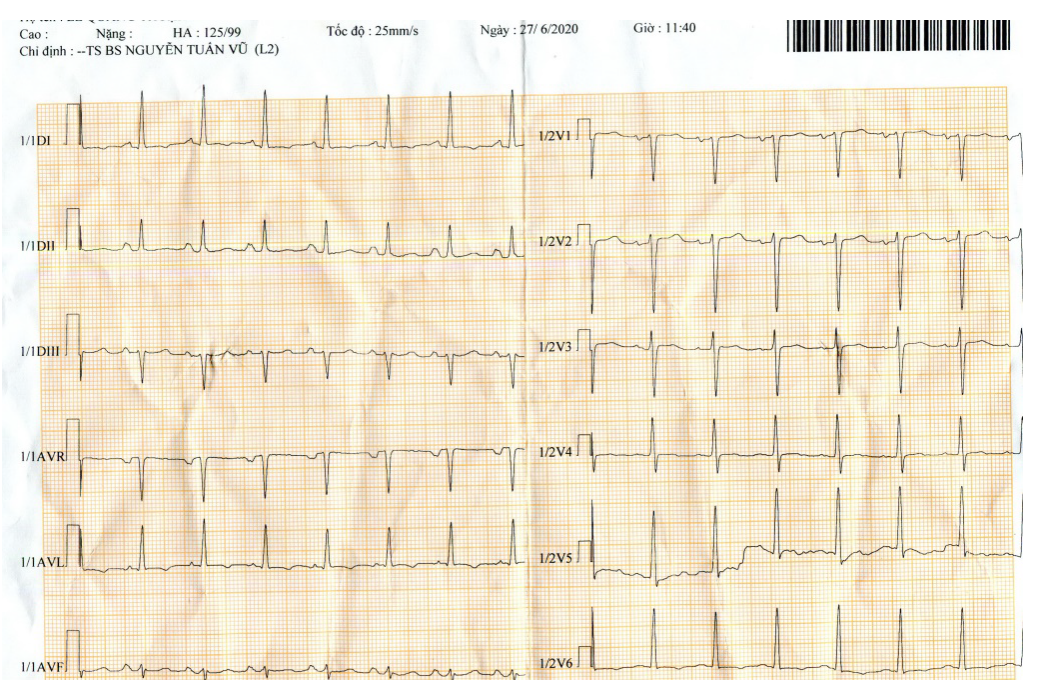

Figure 2. ECG showed sinus tachycardia and LV hypertrophy with Sokolow-Lyon Index $=65 \mathrm{~mm}$

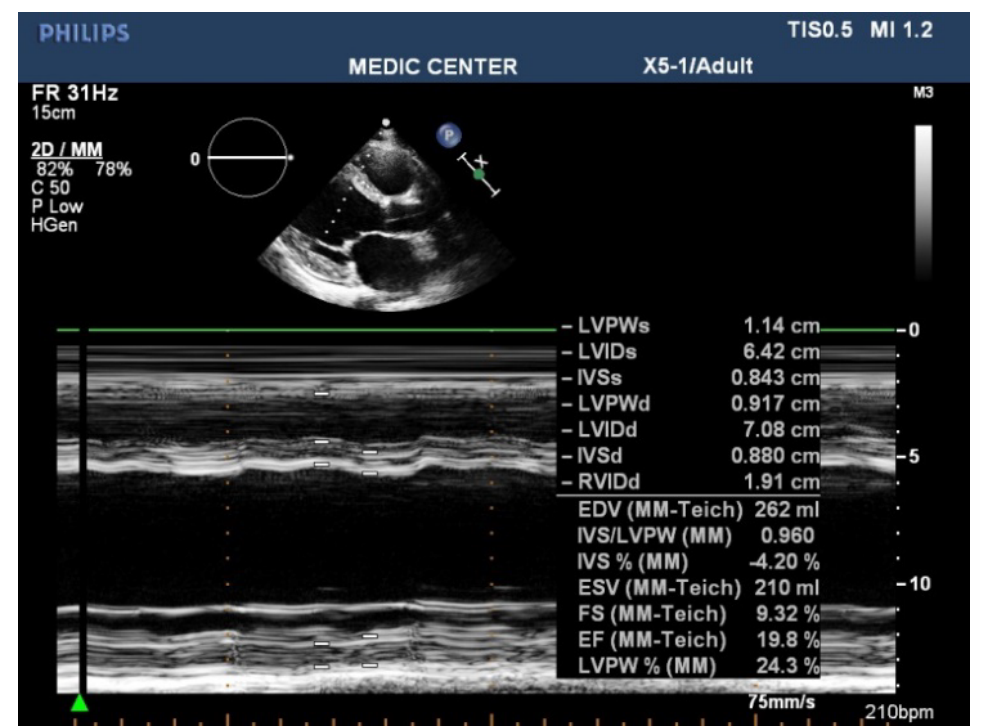

Figure 3. Transthoracic TM mode demonstrating dilated LV with severely reduced global systolic function

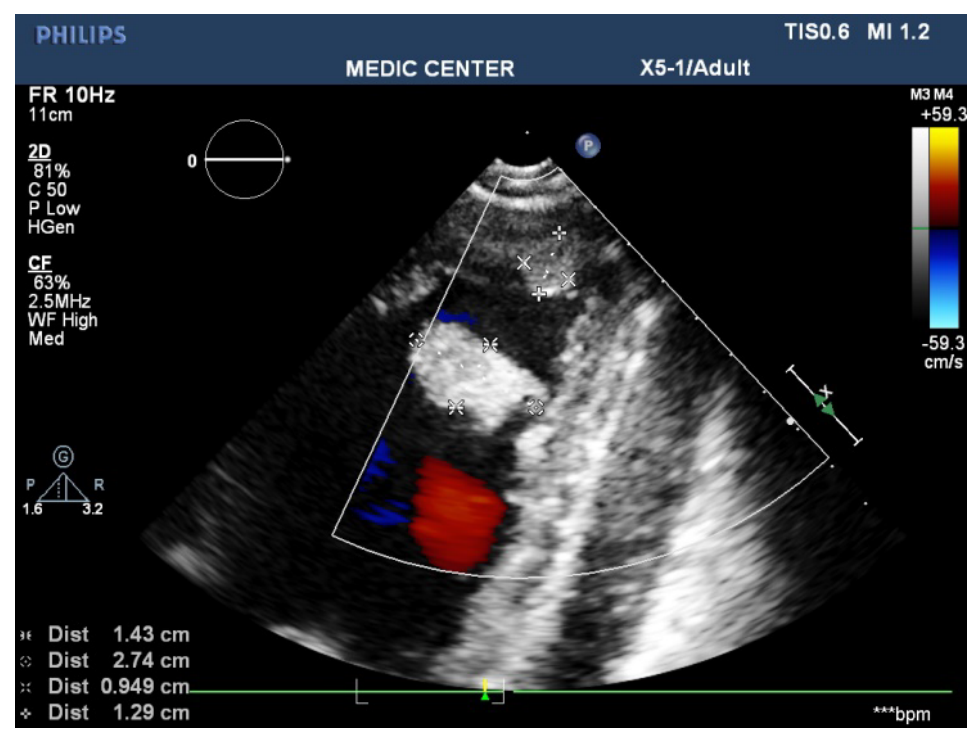

Figure 4. apical longitudinal view showed: a- an apical thrombus 13x 9mm, b-an hyperechogenic mass 27x14 mm with narrow stalk adjacent to posterior IVS 


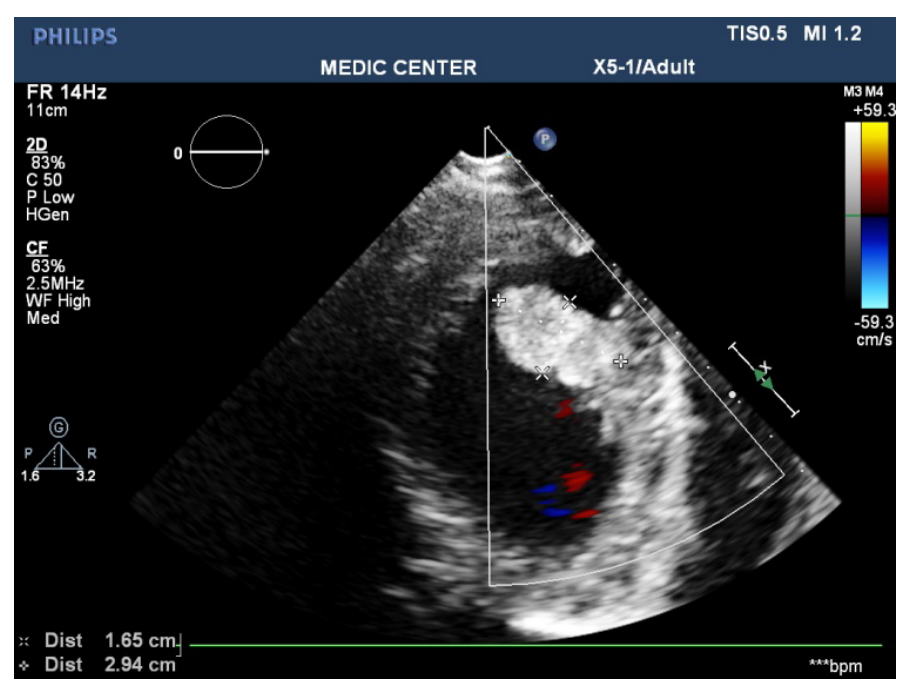

Figure 5. Irregular surface and large stalk hyperechogenic mass attached to IVS illustrated by Two -dimensional Echocardiography

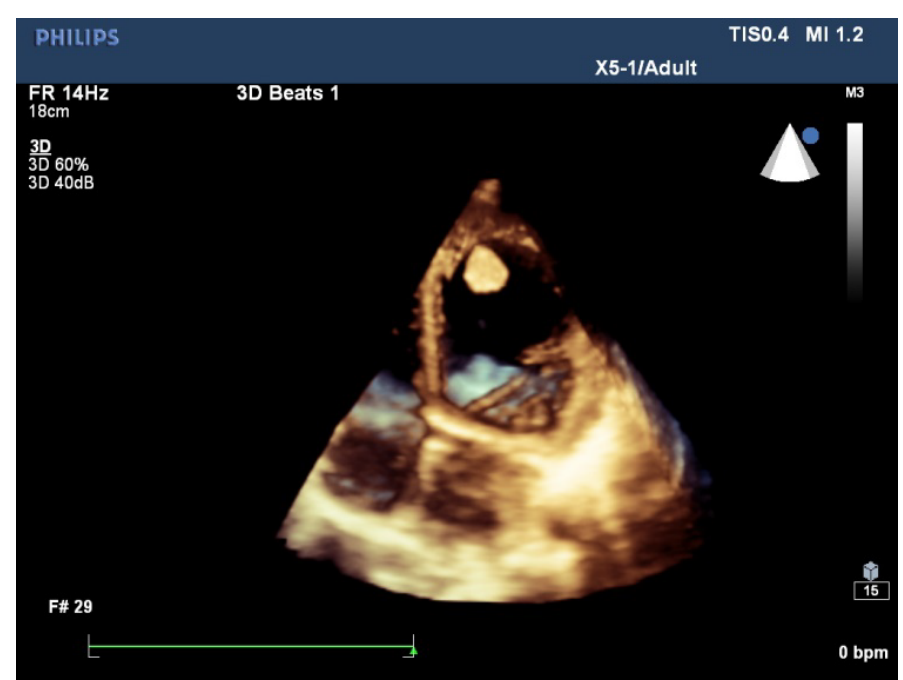

Figure 6. 3DTTE apical $4 \mathrm{C}$ view showed an echogenic mass next to the apex

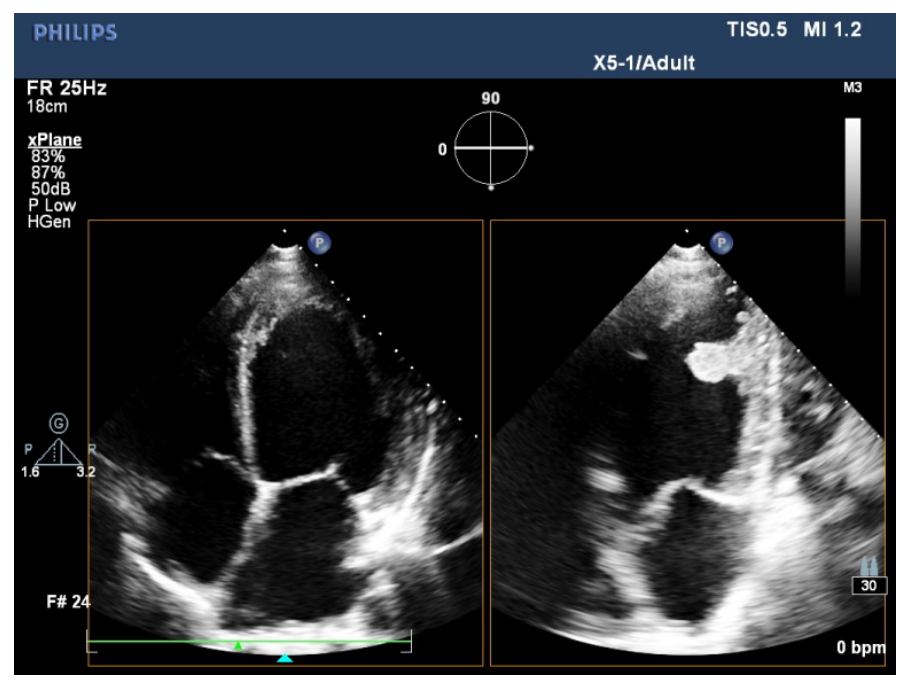

Figure 7. 2D TTE X-plane mode detected the hyperdense echogenic mass inserted to the posterior IVS near the apex

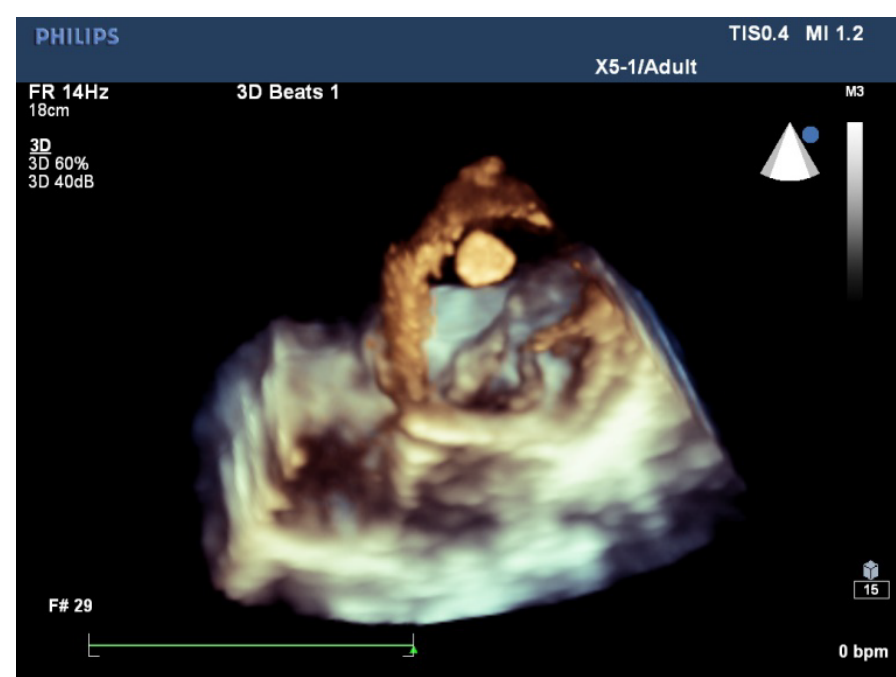

Figure 8. Real-time 3DTTE Live mode viewed from apex to mitral valve, the $3 \mathrm{D}$ image vizualized pedunculated echogenic mass attached next to the apical IVS

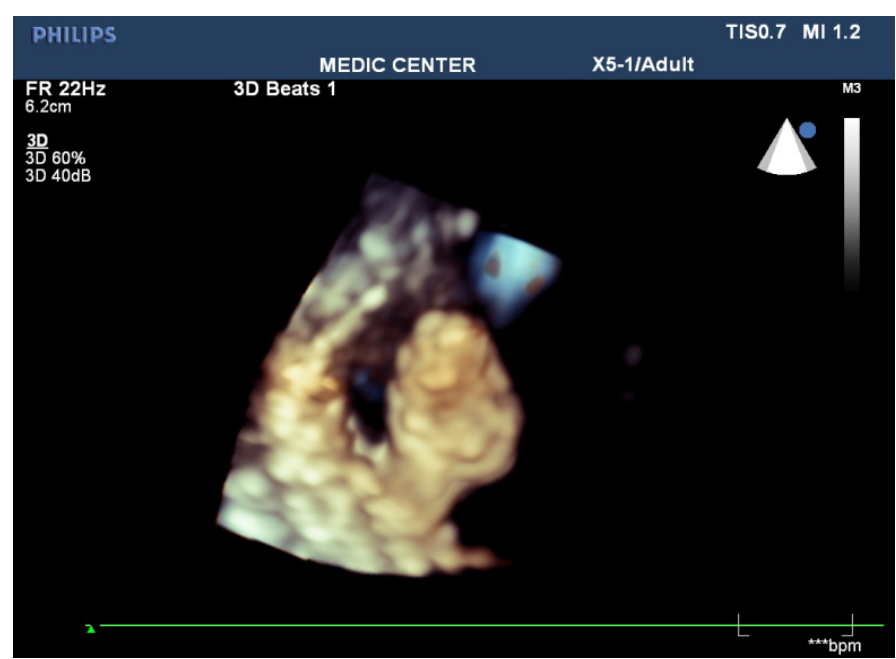

Figure 9. Real-time 3DTTE visualized the echogenic mass that having the narrow pedicle, villious excrescences with high risk of embolic event in compare with polypoid mass

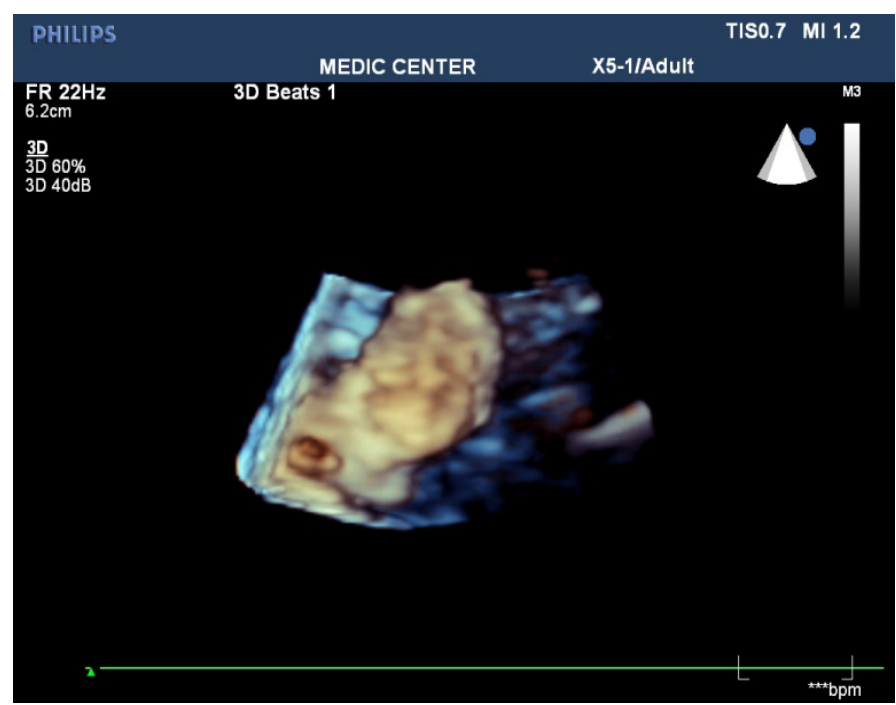

Figure 10. Myxoma viewed from another angle on 3D Zomm mode 
benign and $50 \%$ of them are myxoma.It is estimated that $30-40 \%$ of all myxoma will embolize [1-4].

The majority of myxomas are in the left atrium (75\%), then right atrium $(20 \%)$ and 2,5\% reported for myxoma in left ventricle [4].

Large, mobile, villious excrescences myxomas are more likely embolize than those with polypoid aspect. The risk of embbolic events of our patient surely are very high, but the myxoma cannot resected now. We avoid performing TEE and continue follow up patient with medical treatment of heart failure regarding the severe heart failure of the patient.

Wei Qin, et al. [5] reported a LV myxoma in a an asymptomatic patient 60 year old male, the tumor localated at base of anterolateral papillary muscle detected by $2 \mathrm{D}$ TTE, the tumor was resected suscessfully and no recurrence noted.

Solmaz, et al. [2] reported a large LV myxoma in a 27 year old woman presenting with epigastric pain and weigh loss. This tumor fully filled the LV and compromised the flow of the aortic and mitral valve.The patient was performed 2DTTE then 2DTEE, her tumor originating from LV lateral wall. Surgical procedure was performed and posoperative was without complication.

Hani M. Mahmoud, et al. [3] reported a rare case of big left ventricular myxoma prsenting with a cerebralvascular stroke in a 35 year old male patient. The tumor was detected by routine 2DTTE, Its attached to the inferior septum and has been bulging through the aortic valve. Surgical resection done, his neurological status improved after surgery.

Enrico Natale, et al. reported a case of LV myxoma originating from the interventricular septum and obstructing the left ventricular ouflor tract in a 60 year old woman. The big myxoma was demonstrated by 2DTTE, its attached to the apical portion of the IVS, extending into the left outflow tract and prolapsed through the aortic valve. The myxoma was resected and posoperative was uneventfull.

Occasionally, LV myxoma in my patient was diagnosed unexpectedly because with the the size of myxoma and its location, this tumor did not protrude the mitral orifice like large LA myxoma, but as presented above, this myxoma likely results in cardiac embolic events.

The role of 3D TTE in this case report is the noninvasive imaging method can detecd the myxomas regarding size, location, and especially the $3 \mathrm{D}$ TEE can visualize the high risk findings related to embolic events better the 2DTTE.

\section{Conclusion}

Left ventricle myxoma is rarely seen, patients are commonly are asymptomatic and the tumor may be detected by routine echocardiography.

The patient may be presented at hospital by the cardiac embolic events or hemodynamically compromised symptoms caused by protruding or obstructing the LVOT, aortic and mitral orifices, particularly in case of big LV myxoma.

In majority of cases, LV myxomas are pedunculated and attached to the interventricular septum. The big LV myxomas originating from the base of IVS can obstruct the valvular orifices. The small and apical IVS originating myxoma are usually asymptomatic.

The 3DTTE with advances in image proscessing and display has increased the availability and utility in detecting LV myxoma and providing the the important informations before surgery as well as predicting the risk of embolic events, especially RT-3DTTE records the mobility degree ,the pedicle size and shaped, the location and the villious excrescences of tumor better the 2DTTE used only.

\section{References}

1. Libby Z (2019) Braunwald' Heart Disease. (11th Edn) Elservier, pp: 1869-1871.

2. Fakhari S, Bilehjani E (2016) A large Left Ventricle Myxoma: Presenting with Epigastric Pain and Weight Loss. Case Rep Cardiol.

3. Mahmoud HM, Moursi I (2014) A rare case of a big left ventricular myxoma presenting with a cerebrovascular stroke. The Egyptian Heart J 66: 375-377.

4. Otto C (2017) The Practice of Clinical Echocardiography, 5th edition, Elservier, pp: 843-845.

5. Qin W, Wang L (2014) Left ventricular myxoma: a case report. J Biomed Res 28: 506-508.

Copyright: (C2020 Nguyen Tuan Vu. This is an open-access article distributed under the terms of the Creative Commons Attribution License, which permits unrestricted use, distribution, and reproduction in any medium, provided the original author and source are credited. 